\section{Attitude Estimation Using Biased Gyro and Vector Measurements With Time-Varying Reference Vectors}

Håvard Fjær Grip, Thor I. Fossen, Tor A. Johansen, and Ali Saberi

\begin{abstract}
We present two results on attitude estimation using vector and rate gyro measurements. The first result concerns an observer previously presented by Hamel, Mahony, and Pflimlin, with proven stability results when (i) the reference vectors are stationary; or (ii) the gyro measurements are unbiased. We prove semiglobal stability without either of these assumptions when a parameter projection is added, and convergence from all initial attitudes when using a resetting strategy. The second result is an algorithm for estimation of bias in the body-fixed vector measurements, which is analyzed in combination with the attitude and gyro bias observer.
\end{abstract}

Index Terms-Navigation, estimation

\section{INTRODUCTION}

When determining the attitude of an object with respect to a reference frame, it is common to use a set of vectors measured in a body-fixed coordinate frame-using sensors such as magnetometers, accelerometers, or sun sensors-and a corresponding set of reference vectors in the reference frame. The attitude can be resolved by comparing these vectors using a number of different algorithms, such as TRIAD and QUEST, provided at least two non-parallel vector measurements are available (see [1], [2]). Vector measurements are typically affected by noise, which is particularly significant at high frequencies. It is therefore common to combine vector measurements with measurements from rate gyroscopes, which complement the vector measurements by providing high-frequency attitude information.

Most commonly, vector and gyro measurements are integrated using an extended Kalman filter (EKF). An overview of early EKFs is given by Lefferts, Markley, and Shuster [3], whereas Crassidis, Markley, and Cheng [4] survey more recent results using EKFs as well as other estimation techniques. Integrated inertial (INS) and satellite (GNSS) navigation systems have also been studied for several decades and are typically based on EKFs [5], [6]. Such systems implicitly determine the attitude by matching the accelerometer vector with changes in the velocity vector. Measurements are also affected by other errors, such as bias, alignment, and scale-factor errors. These are often counteracted by including additional parameters in the estimation algorithm.

One alternative to the EKF is to construct nonlinear observers with explicitly proven stability properties. Such observers typically have a smaller computational footprint than the EKF, and they are therefore of particular interest as low-cost navigation equipment becomes available in a wide range of products, such as cell phones, cars, and small unmanned vehicles. The first observer of this type was presented by Salcudean [7]. It was later extended by Vik and Fossen [8] by adding gyro bias and linear velocity estimation in the context of GPS/INS integration. Thienel and Sanner [9] improved the stability analysis by deriving a uniform complete observability argument that guarantees exponentially vanishing estimation errors (including bias). The nonlinear observers based on Salcudean's work assume that the attitude is algebraically resolved in a separate subsystem and provided as a measurement to the observer. A drawback of this

H. F. Grip and A. Saberi are with the School of Electrical Engineering and Computer Science, Washington State University, Pullman, WA 99164 2752, USA. The work of H. F. Grip is supported by the Research Council of Norway. The work of A. Saberi is partially supported by NAVY grants ONR KKK777SB001 and ONR KKK760SB0012.

T. I. Fossen and T. A. Johansen are with the Department of Engineering Cybernetics, Norwegian University of Science and Technology, O.S. Bragstads plass 2D, 7491 Trondheim, Norway. assumption is that the noise characteristics of the resolved attitude may differ significantly from the characteristics of the measurements used to produce it. For example, the influence of vector noise varies depending on how well-conditioned the attitude resolution problem is at any given point in time. The separate attitude resolution also adds computational complexity (see also discussion in [10]).

More recently, Hamel and Mahony [11] and Mahony, Hamel, and Pflimlin [10] have proposed an observer that does not rely on separate attitude resolution, but instead makes use of vector measurements directly. Their observer includes estimation of gyro bias and is rigorously analyzed with respect to stability. An underlying assumption, however, is that the reference vectors are stationary. Stability in the case of time-varying reference vectors is studied in a later paper by Mahony, Hamel, Trumpf, and Lageman [12] and in a recent paper by Hua [13], albeit without considering gyro bias estimation. It is proven that the observer without bias estimation can handle timevarying reference vectors as well. Moreover, Hua presents algorithms for using the derivative of GNSS velocity to form a reference vector without explicit differentiation.

Mahony et al. [12] also considered attitude estimation using a single vector measurement, which is sufficient if a persistency-ofexcitation (PE) condition is satisfied. This problem has previously been considered by Kinsey and Whitcomb [14] and Lee, Leok, McClamroch, and Sanyal [15], also without considering gyro bias. Vasconcelos, Silvestre, and Oliveira [16] have considered simultaneous attitude and gyro bias estimation by realigning the measurements using, for example, a singular value decomposition based on the matrix of reference vectors. Globally exponentially stable attitude estimation based on time-varying reference vectors-by estimating a full $3 \times 3$ rotation matrix that is not confined to $\mathrm{SO}(3)$ - has recently been considered by Batista, Silvestre, and Oliveira [17], [18] and Grip, Saberi, and Johansen [19], [20], albeit without bias estimation.

\section{A. Goals and Contributions}

The ability to handle time-varying reference vectors is an important one, in particular, when accelerometers are used to provide vector measurements. In applications with slow dynamics, the gravity vector can be used as a stationary reference, but in general the true reference vector is time-varying. Some compensation for vehicle acceleration is possible by using, for example, additional measurements, a vehicle model, low-pass filtering, or compensation of Coriolis terms. On the other hand, if GNSS measurements are available, then the actual reference vector is implicitly available via the derivative of GNSS velocity. This scenario is the main motivation for this note.

We focus on two specific problems regarding attitude and bias estimation. First, we consider the observer presented by Hamel and Mahony [11] and Mahony et al. [10]. This observer has proven stability properties when either (i) the reference vectors are stationary; or (ii) the reference vectors are time-varying but the gyro measurements are bias-free. What is not considered by Hamel and Mahony [11], Mahony et al. [10], [12], or Hua [13] is the case when the reference vectors are time-varying and the gyro measurements are biased. By adding a parameter projection to the bias estimation, we show that the observer is applicable in this case as well; in particular, we prove semiglobal exponential stability, and we show how attractivity from arbitrary initial attitudes can be guaranteed by an additional resetting scheme.

In addition to gyro bias, bias in the vector measurements can constitute a significant problem. For example, low-cost accelerometers typically have a large bias; on the other hand, the corresponding reference vector, derived using the gravity vector and possibly the derivative of GNSS velocity, is typically subject to a very small bias. 
Motivated by this situation, we present an exponentially converging algorithm for estimating bias in the body-fixed vector measurement by using a bias-free reference vector.

A conference version of this paper, without the results based on resetting and physically realistic examples, was recently presented at the IFAC World Congress [21].

\section{PRELIMINARIES}

Any rotation can be described by a unit-length rotation axis $k \in \mathbb{R}^{3}$ and a rotation angle $\beta$. The corresponding unit quaternion is defined as $q=\left[s, r^{\top}\right]^{\top}=\left[\cos (\beta / 2), k^{\top} \sin (\beta / 2)\right]^{\top}$, which implies $\|q\|^{2}=s^{2}+\|r\|^{2}=1$. We shall use both quaternions and rotation matrices $R \in \mathrm{SO}(3)$ to represent rotations. The two representations are related by $R=R_{q}(s, r):=I+2 s S(r)+2 S(r)^{2}$, where $S(x)$ denotes the skew-symmetric matrix such that $S(x) y=x \times y$. We denote by $\|\cdot\|$ the Euclidean norm for vectors in $\mathbb{R}^{n}$ and the Frobenius norm for matrices in $\mathbb{R}^{m \times m}$. For $(X, x) \in \mathbb{R}^{m \times m} \times \mathbb{R}^{n}$, we define $\|(X, x)\|=\sqrt{\|X\|^{2}+\|x\|^{2}}$. Throughout the note, we assume that all dynamical systems are initialized at time $t=0$. For time-varying quantities, we usually omit the time argument.

\section{Problem Formulation}

Let $R \in \mathrm{SO}(3)$ denote the rotation matrix from the body-fixed frame to an inertial reference frame. The rotation matrix behaves according to the kinematic equation

$$
\dot{R}=R S(\omega),
$$

where $\omega$ is the angular velocity in body-fixed coordinates, bounded by $\|\omega\| \leq \bar{\omega}$. A measurement $\omega_{m}=\omega+b_{g}$ is available, where $b_{g}$ represents gyro bias. We assume that $b_{g}$ is constant and belongs to an a priori known, compact, convex set $\mathscr{B}_{g} \subset \mathbb{R}^{3}$. We furthermore assume availability of $n$ vectors $v_{j}^{b}, j \in 1, \ldots, n$, in the body-fixed frame, and corresponding reference vectors $v_{j}^{i}=R v_{j}^{b}$ in the inertial frame. The reference vectors are presumed to be time-varying but bounded away from zero; that is $\left\|v_{j}^{i}\right\| \geq \underline{c} \geq 0$. For simplicity we introduce normalized versions of these vectors as $\bar{v}_{j}^{b}=v_{j}^{b} /\left\|v_{j}^{b}\right\|$ and $\bar{v}_{j}^{i}=v_{j}^{i} /\left\|v_{j}^{i}\right\|$. As in other places in the literature [12], [13], we ensure observability through an additional assumption that requires there to always be two normalized reference vectors with the angle between them bounded away from $0^{\circ}$ and $180^{\circ}$.

Assumption 1: There exists a constant $c_{\mathrm{obs}}>0$ such that, for each $t \geq 0$, there are indices $j, k \in 1, \ldots, n$ such that $\left\|\bar{v}_{j}^{i} \times \bar{v}_{k}^{i}\right\| \geq c_{\text {obs }}$.

The estimate of the rotation matrix will be denoted by $\hat{R} \in \mathrm{SO}(3)$, and the estimation error will be represented by $\tilde{R}:=R \hat{R}^{\top} \in \mathrm{SO}(3)$. The goal is to ensure that $\tilde{R} \rightarrow I$ as $t \rightarrow \infty$, which implies that $\hat{R}$ asymptotically represents the same rotation as $R$. The attitude error will also be represented by a unit quaternion $\tilde{q}=\left[\tilde{s}, \tilde{r}^{\top}\right]^{\top}$ corresponding to $\tilde{R}$. Note that $\tilde{R}=I$ is equivalent to $|\tilde{s}|=1$ and $\tilde{r}=0$. It is also useful to note that $\tilde{s}=0$ corresponds to a rotation error of $180^{\circ}$ about some axis (i.e., the maximal error rotation angle).

\section{Previous Results}

Hamel and Mahony [11] and Mahony et al. [10] present the following observer for estimating $R$ and $b_{g}$ :

$$
\begin{aligned}
\dot{\hat{R}} & =\hat{R} S\left(\omega_{m}-\hat{b}_{g}+\sigma\right), \\
\dot{\hat{b}}_{g} & =-k_{I} \sigma, \\
\text { where } \sigma & =\sum_{j=1}^{n} k_{j} \bar{v}_{j}^{b} \times \hat{R}^{\top} \bar{v}_{j}^{i},
\end{aligned}
$$

with $k_{j} \geq k_{P}>0, j=1, \ldots, n$, as well as $k_{I}>0$, representing observer gains. Under the assumption that the reference vectors $\bar{v}_{j}^{i}, j=1, \ldots, n$, are stationary, it is proven that the observer error dynamics is locally exponentially stable and that the observer states converge to the true states for almost all initial conditions. If the reference vectors $\bar{v}_{j}^{i}$, $j=1, \ldots, n$, are time-varying, then convergence can still be proven if gyro bias is disregarded (i.e., $\omega_{m}=\omega$ ). This can be done, for example, based on the analysis of Hua [13], as outlined in the remainder of this section.

The dynamics of $\tilde{R}$ is described by $\dot{\tilde{R}}=\dot{R} \hat{R}^{\top}+R \dot{\hat{R}}^{\top}=R S(\omega) \hat{R}^{\top}$ $R S\left(\omega_{m}+\sigma\right) \hat{R}^{\top}=-R S(\sigma) \hat{R}^{\top}=-S(R \sigma) \tilde{R}$, where $S(x)^{\top}=-S(x)$ and $R S(x) R^{\top}=S(R x)$ have been used. Note that $\tilde{R}$ is confined to $\mathrm{SO}(3)$. The dynamics described by a corresponding unit quaternion is [22]

$$
\begin{aligned}
& \dot{\tilde{s}}=\frac{1}{2} \tilde{r}^{\top} R \sigma, \\
& \dot{\tilde{r}}=-\frac{1}{2}(\tilde{s} I-S(\tilde{r})) R \sigma .
\end{aligned}
$$

The unit quaternion dynamics is confined to the unit sphere; that is, $\tilde{s}^{2}+\|\tilde{r}\|^{2}=1$. Note that

$$
\begin{aligned}
R \sigma & =\sum_{j=1}^{n} k_{j} R S\left(\bar{v}_{j}^{b}\right) \hat{R}^{\top} \bar{v}_{j}^{i} \\
& =\sum_{j=1}^{n} k_{j} S\left(R \bar{v}_{j}^{b}\right) \tilde{R} \bar{v}_{j}^{i}=\sum_{j=1}^{n} k_{j} S\left(\bar{v}_{j}^{i}\right)\left(I+2 \tilde{S} S(\tilde{r})+2 S(\tilde{r})^{2}\right) \bar{v}_{j}^{i} \\
& =2 \sum_{j=1}^{n} k_{j} S\left(\bar{v}_{j}^{i}\right)\left(\tilde{s} S(\tilde{r})+S(\tilde{r})^{2}\right) \bar{v}_{j}^{i}
\end{aligned}
$$

By defining the Lyapunov-like function $V(\tilde{s})=1-\tilde{s}^{2}=\|\tilde{r}\|^{2}$, one obtains the derivative

$$
\begin{aligned}
\dot{V} & =-2 \tilde{s} \tilde{r}^{\top} \sum_{j=1}^{n} k_{j} S\left(\bar{v}_{j}^{i}\right)\left(\tilde{s} S(\tilde{r})+S(\tilde{r})^{2}\right) \bar{v}_{j}^{i} \\
& =-2 \tilde{s} \sum_{j=1}^{n} k_{j}\left(\tilde{r} \times \bar{v}_{j}^{i}\right)^{\top}(\tilde{s} I+S(\tilde{r}))\left(\tilde{r} \times \bar{v}_{j}^{i}\right) \leq-2 k_{P} \sum_{j=1}^{n} \tilde{s}^{2}\left\|\tilde{r} \times \bar{v}_{j}^{i}\right\|^{2}
\end{aligned}
$$

where we have used the identity $y^{\top} S(x) y=0$. Using the inequality $\left\|\tilde{r} \times \bar{v}_{j}^{i}\right\|^{2}+\left\|\tilde{r} \times \bar{v}_{k}^{i}\right\|^{2} \geq \frac{1}{2}\|\tilde{r}\|^{2}\left\|\bar{v}_{j}^{i} \times \bar{v}_{k}^{i}\right\|^{2}$ (see [13]) together with Assumption 1 yields $\dot{V} \leq-k_{P} c_{\mathrm{obs}}^{2} \tilde{s}^{2}\|\tilde{r}\|^{2}=-k_{P} c_{\mathrm{obs}}^{2} \tilde{s}^{2}\left(1-\tilde{s}^{2}\right)$. Since $\dot{V}$ is negative whenever $\tilde{s} \neq 0$ and $|\tilde{s}| \neq 1$, it is straightforward to show that for any initial condition satisfying $\tilde{R}(0) \in\left\{R_{q}(\tilde{s}, \tilde{r})|| \tilde{s} \mid>0\right\}$ (i.e., the initial rotation error is strictly less than $180^{\circ}$ ), $|\tilde{s}|$ increases monotonically such that $|\tilde{s}| \rightarrow 1$ (implying $\|\tilde{r}\| \rightarrow 0$ and $\tilde{R} \rightarrow I$ ).

\section{Attitude AND Gyro Bias Estimation with TIME-VARYING REFERENCE VECTORS}

We now build on the analysis outlined in the previous section to show that the observer, including bias estimation, is applicable even if the reference vectors are time-varying. The only modification that we make to (2) is to add a parameter projection of the type shown in the Appendix of this note (recalled from [23, App. E]), which ensures that $\hat{b}_{g}$ remains within a compact, convex set $\hat{\mathscr{B}}_{g} \supset \mathscr{B}_{g}$, defined slightly larger than $\mathscr{B}_{g}$. Accordingly, we redefine $(2 b)$ as

$$
\dot{\hat{b}}_{g}=\operatorname{Proj}\left(\hat{b}_{g},-k_{I} \sigma\right) \text {. }
$$

Defining the bias estimation error $\tilde{b}_{g}=b_{g}-\hat{b}_{g}$, we obtain the complete error dynamics

$$
\begin{gathered}
\dot{\tilde{R}}=-S\left(R \tilde{b}_{g}+R \sigma\right) \tilde{R}, \\
\dot{\tilde{b}}_{g}=-\operatorname{Proj}\left(\hat{b}_{g},-k_{I} \sigma\right) .
\end{gathered}
$$

We define a parameterized set $\tilde{\mathscr{R}}(\varepsilon)=\left\{R_{q}(\tilde{s}, \tilde{r})|| \tilde{s} \mid \geq \varepsilon\right\}$, where $0<\varepsilon<1$.

Theorem 1: Consider the dynamics of the error $\left(\tilde{R}, \tilde{b}_{g}\right) \in \mathrm{SO}(3) \times$ $\mathbb{R}^{3}$, described by (5). For each $0<\varepsilon<1$, there exists a $\bar{k}_{P}>0$ such 
that, for all $k_{P}>\bar{k}_{P}$, the equilibrium point $(I, 0)$ is exponentially stable with all initial conditions such that $\tilde{R}(0) \in \tilde{\mathscr{R}}(\varepsilon)$ and $\hat{b}_{g}(0) \in \hat{\mathscr{B}}_{g}$ contained in the region of attraction.

Proof: Let $M$ be a bound on $\left\|\tilde{b}_{g}\right\|$ on the compact set $\tilde{\mathscr{B}}_{g}=$ $\left\{b_{g}-\hat{b}_{g} \mid b_{g} \in \mathscr{B}_{g}, \hat{b}_{g} \in \hat{\mathscr{B}}_{g}\right\}$. We start by defining $\ell>0$ such that $\ell<\min \left\{1 /\left(2 k_{I}\right), c_{\mathrm{obs}}^{2} \varepsilon^{2} /\left(12 n M+8 k_{I} n\right)\right\}$. Next, define $a=c_{\mathrm{obs}}^{2} \varepsilon^{2}-$ $\ell\left(12 n M+8 k_{I} n\right)$ (note that $\left.a>0\right)$ and let $\bar{k}_{P}=\max \left\{M /\left(c_{\mathrm{obs}}^{2} \varepsilon^{2}(1-\right.\right.$ $\left.\left.\left.\varepsilon^{2}\right)\right),\left((1+2 \ell \bar{\omega})^{2}+4 \ell^{2} M^{2} \varepsilon^{2}\right) /\left(4 a \ell \varepsilon^{2}\right)\right\}$. Using unit quaternions the error dynamics becomes

$$
\begin{aligned}
\dot{\tilde{s}} & =\frac{1}{2} \tilde{r}^{\top} R\left(\tilde{b}_{g}+\sigma\right), \\
\dot{\tilde{r}} & =-\frac{1}{2}(\tilde{s} I-S(\tilde{r})) R\left(\tilde{b}_{g}+\sigma\right), \\
\dot{\tilde{b}}_{g} & =-\operatorname{Proj}\left(\hat{b}_{g},-k_{I} \sigma\right) .
\end{aligned}
$$

We know that $\hat{b}_{g}(0) \in \hat{\mathscr{B}}_{g}$. Thus, for all all $t \geq 0, \hat{b}_{g}(t) \in \hat{\mathscr{B}}_{g}$, and hence $\tilde{b}_{g}(t) \in \tilde{\mathscr{B}}_{g}$. Consider the derivative of $V$ defined in Section IV:

$$
\dot{V} \leq-\tilde{s} \tilde{r}^{\top} R \tilde{b}_{g}-k_{P} c_{\mathrm{obs}}^{2} \tilde{s}^{2}\left(1-\tilde{s}^{2}\right) \leq M-k_{P} c_{\mathrm{obs}}^{2} \tilde{s}^{2}\left(1-\tilde{s}^{2}\right) .
$$

Using the property $k_{P}>\bar{k}_{P} \geq M /\left(c_{\mathrm{obs}}^{2} \varepsilon^{2}\left(1-\varepsilon^{2}\right)\right)$, we find that for $|\tilde{s}|=\varepsilon$, we have $\dot{V} \leq M-k_{P} c_{\text {obs }}^{2} \varepsilon^{2}\left(1-\tilde{\varepsilon}^{2}\right)<M-M=0$. This implies that $V$ is strictly decreasing whenever $|\tilde{s}|=\varepsilon$, which in turn implies that $|\tilde{S}|$ is strictly increasing. It follows from $|\tilde{s}(0)| \geq \varepsilon$ and continuity of the solutions that $|\tilde{s}|$ can never become smaller than $\varepsilon$, and we therefore assume $|\tilde{s}| \geq \varepsilon$ in the remainder of the analysis.

Consider the Lyapunov-like function

$$
W\left(\tilde{r}, \tilde{s}, \tilde{b}_{g}\right)=V(\tilde{s})+2 \ell \tilde{s} \tilde{r}^{\top} R \tilde{b}_{g}+\frac{\ell}{2 k_{I}} \tilde{b}_{g}^{\top} \tilde{b}_{g} .
$$

We have $W \geq\|\tilde{r}\|^{2}-2 \ell\|\tilde{r}\|\left\|\tilde{b}_{g}\right\|+\frac{\ell}{2 k_{l}}\left\|\tilde{b}_{g}\right\|^{2}$, where we have used the fact that $|\tilde{s}| \leq 1$. This quadratic expression is positive definite with respect to $\tilde{r}$ and $\tilde{b}_{g}$ due to the bound on $\ell$ described above. It follows that there exist positive constants $\alpha_{1}$ and $\alpha_{2}$ such that $\alpha_{1}\left\|\left(\tilde{r}, \tilde{b}_{g}\right)\right\|^{2} \leq W \leq \alpha_{2}\left\|\left(\tilde{r}, \tilde{b}_{g}\right)\right\|^{2}$. For the time derivative of $W$ we calculate

$$
\begin{aligned}
\dot{W} \leq & -\tilde{s} \tilde{r}^{\top} R \tilde{b}_{g}-k_{P} c_{\mathrm{obs}}^{2} \tilde{s}^{2}\left(1-\tilde{s}^{2}\right)+\ell \tilde{r}^{\top} R \tilde{b}_{g} \tilde{r}^{\top} R \tilde{b}_{g} \\
& +\ell \tilde{r}^{\top} R \sigma \tilde{r}^{\top} R \tilde{b}_{g}-\ell \tilde{s}^{2} \tilde{b}_{g}^{\top} \tilde{b}_{g} \\
& -\ell \sigma^{\top} R^{\top}\left(\tilde{s}^{2} I+\tilde{s} S(\tilde{r})\right) R \tilde{b}_{g}+2 \ell \tilde{s} \tilde{r}^{\top} R S(\omega) \tilde{b}_{g} \\
& -2 \ell \tilde{s} \tilde{r}^{\top} R \operatorname{Proj}\left(\hat{b}_{g},-k_{I} \sigma\right)-\frac{\ell}{k_{I}} \tilde{b}_{g}^{\top} \operatorname{Proj}\left(\hat{b}_{g},-k_{I} \sigma\right) \\
\leq & \|\tilde{r}\|\left\|\tilde{b}_{g}\right\|-k_{P} c_{\mathrm{obs}} \tilde{s}^{2}\|\tilde{r}\|^{2}+\ell\|\tilde{r}\|^{2}\left\|\tilde{b}_{g}\right\|^{2}-\ell \tilde{s}^{2}\left\|\tilde{b}_{g}\right\|^{2} \\
& -\ell \sigma^{\top} R^{\top}\left(\left(1-\|\tilde{r}\|^{2}\right) I+\tilde{s} S(\tilde{r})-\tilde{r} \tilde{r}^{\top}\right) R \tilde{b}_{g} \\
& +2 \ell \bar{\omega}\|\tilde{r}\|\left\|\tilde{b}_{g}\right\|+2 \ell k_{I}\|\tilde{r}\|\|\sigma\|+\ell \sigma^{\top} \tilde{b}_{g} \\
= & \|\tilde{r}\|\left\|\tilde{b}_{g}\right\|-k_{P} c_{\mathrm{obs}} \tilde{s}^{2}\|\tilde{r}\|^{2}+\ell\|\tilde{r}\|^{2}\left\|\tilde{b}_{g}\right\|^{2}-\ell \tilde{s}^{2}\left\|\tilde{b}_{g}\right\|^{2} \\
& -\ell \sigma^{\top} R^{\top}\left(-\|\tilde{r}\|^{2} I+\tilde{s} S(\tilde{r})-\tilde{r} \tilde{r}^{\top}\right) R \tilde{b}_{g} \\
& +2 \ell \bar{\omega}\|\tilde{r}\|\left\|\tilde{b}_{g}\right\|+2 \ell k_{I}\|\tilde{r}\|\|\sigma\|,
\end{aligned}
$$

where we have used the properties that $\left\|\operatorname{Proj}\left(\hat{b}_{g},-k_{I} \sigma\right)\right\| \leq k_{I}\|\sigma\|$ and $-\tilde{b}_{g}^{\top} \operatorname{Proj}\left(\hat{b}_{g},-k_{I} \sigma\right) \leq k_{I} \tilde{b}_{g}^{\top} \sigma[23$, Lemma E.1]. Note that $\|\sigma\|=$ $\|R \sigma\|=2\left\|\sum_{j=1}^{n} k_{j} S\left(\bar{v}_{j}^{i}\right)\left(\tilde{s} S(\tilde{r})+S(\tilde{r})^{2}\right) \bar{v}_{j}^{i}\right\| \leq 4 k_{P} n\|\tilde{r}\|$. Using this bound, as well as $|\tilde{s}| \geq \varepsilon$, we obtain

$$
\begin{aligned}
\dot{W} \leq & \|\tilde{r}\|\left\|\tilde{b}_{g}\right\|-k_{P} c_{\mathrm{obs}}^{2} \varepsilon^{2}\|\tilde{r}\|^{2}+\ell\|\tilde{r}\|^{2}\left\|\tilde{b}_{g}\right\|^{2}-\ell \varepsilon^{2}\left\|\tilde{b}_{g}\right\|^{2} \\
& +4 \ell k_{P} n\|\tilde{r}\|\left(2\|\tilde{r}\|^{2}+\|\tilde{r}\|\right)\left\|\tilde{b}_{g}\right\|+2 \ell \bar{\omega}\|\tilde{r}\|\left\|\tilde{b}_{g}\right\|+8 \ell k_{I} k_{P} n\|\tilde{r}\|^{2} \\
\leq & \|\tilde{r}\|\left\|\tilde{b}_{g}\right\|-k_{P} c_{\mathrm{obs}}^{2} \varepsilon^{2}\|\tilde{r}\|^{2}+\ell M^{2}\|\tilde{r}\|^{2}-\ell \varepsilon^{2}\left\|\tilde{b}_{g}\right\|^{2} \\
& +12 \ell k_{P} n M\|\tilde{r}\|^{2}+2 \ell \bar{\omega}\|\tilde{r}\|\left\|\tilde{b}_{g}\right\|+8 \ell k_{I} k_{P} n\|\tilde{r}\|^{2} \\
= & -\left[\begin{array}{ll}
\|\tilde{r}\| & \left.\left\|\tilde{b}_{g}\right\|\right]
\end{array}\right]\left[\begin{array}{cc}
k_{P} a-\ell M^{2} & -\frac{1}{2}(1+2 \ell \bar{\omega}) \\
-\frac{1}{2}(1+2 \ell \bar{\omega}) & \ell \varepsilon^{2}
\end{array}\right]\left[\begin{array}{c}
\|\tilde{r}\| \\
\left\|\tilde{b}_{g}\right\|
\end{array}\right] .
\end{aligned}
$$

Inspecting the first-order principal minor of the above matrix, we have $k_{P} a-\ell M^{2}>\bar{k}_{P} a-\ell M^{2} \geq\left((1+2 \ell \bar{\omega})^{2}+\right.$ $\left.\left.4 \ell^{2} M^{2} \varepsilon^{2}\right)\right) a /\left(4 a \ell \varepsilon^{2}\right)-\ell M^{2} \geq 4 \ell^{2} M^{2} \varepsilon^{2} a /\left(4 a \ell \varepsilon^{2}\right)-\ell M^{2}=$ $\ell M^{2}-\ell M^{2}=0$. For the second-order principal minor, we have $\left(k_{P} a-\ell M^{2}\right) \ell \varepsilon^{2}-\frac{1}{4}(1+2 \ell \bar{\omega})^{2}>\left(\bar{k}_{P} a-\ell M^{2}\right) \ell \varepsilon^{2}-\frac{1}{4}(1+2 \ell \bar{\omega})^{2} \geq$ $\left(\left((1+2 \ell \bar{\omega})^{2}+4 \ell^{2} M^{2} \varepsilon^{2}\right) a /\left(4 a \ell \varepsilon^{2}\right)-\ell M^{2}\right) \ell \varepsilon^{2}-\frac{1}{4}(1+2 \ell \bar{\omega})^{2}=$ $\left((1+2 \ell \bar{\omega})^{2} a /\left(4 a \ell \varepsilon^{2}\right)+\ell M^{2}-\ell M^{2}\right) \ell \varepsilon^{2}-\frac{1}{4}(1+2 \ell \bar{\omega})^{2}=$ $\frac{1}{4}(1+2 \ell \bar{\omega})^{2}-\frac{1}{4}(1+2 \ell \bar{\omega})^{2}=0$. Since both principal minors are positive, the matrix is positive definite, which implies that there exists an $\alpha_{3}>0$ such that $\dot{W} \leq-\alpha_{3}\left\|\left(\tilde{r}, \tilde{b}_{g}\right)\right\|^{2}$. Hence, there is an $\alpha>0$ such that $\dot{W} \leq-\alpha W$. Using the comparison lemma [24, Lemma 3.4], we conclude that there exist positive constants $K$ and $\lambda$ such that for all $t \geq 0$, $\left\|\left(\tilde{r}(t), \tilde{b}_{g}(t)\right)\right\| \leq K \mathrm{e}^{-\lambda t}\left\|\left(\tilde{r}(0), \tilde{b}_{g}(0)\right)\right\|$. Using $\|I-\tilde{R}\|=\sqrt{8}\|\tilde{r}\|$, we therefore have $\left\|\left(I-\tilde{R}(t), \tilde{b}_{g}(t)\right)\right\| \leq \sqrt{8} K \mathrm{e}^{-\lambda t}\left\|\left(I-\tilde{R}(0), \tilde{b}_{g}(0)\right)\right\|$.

Theorem 1 specifies that the initial error rotation angle must be smaller than $180^{\circ}$ by a certain margin, which can be arbitrarily reduced by increasing $k_{P}$. The bias estimate must be initialized from the set $\hat{\mathscr{B}}_{g}$, which can be chosen arbitrarily large. The stability result is therefore best characterized as semiglobal (in the same way as [13, Th. 1]), which is slightly weaker than the almost-global result for stationary reference vectors [10].

According to the stability result, there is a possibility of the attitude estimate becoming stuck if the initial error angle is close to $180^{\circ}$. Although simulation results indicate that this is not a practical problem, we nevertheless wish to guarantee convergence for all initial attitudes. One way to achieve this is to check the objective function $J(\hat{R})=\frac{1}{2} \sum_{j=1}^{n}\left\|\bar{v}_{j}^{b}-\hat{R}^{\top} \bar{v}_{j}^{i}\right\|^{2}$, proposed by Wahba [1], periodically at times $\tau, 2 \tau, 3 \tau, \ldots$, where $\tau>0$ is a design variable. If $J(\hat{R})>\delta$, where $\delta>0$ is another design variable, then $\hat{R}$ is reset to the attitude $R^{*}$, which is computed based on the vector measurements by using an algorithm such as TRIAD or QUEST (theoretically yielding $R^{*}=R$ ).

Theorem 2: Suppose that the semiglobally stabilizing observer is tuned according to Theorem 1, such that, for some choice of $0<$ $\varepsilon<1$, all initial conditions with $\tilde{R}(0) \in \tilde{\mathscr{R}}(\varepsilon)$ and $\hat{b}_{g}(0) \in \hat{\mathscr{B}}_{g}$ are contained in the region of attraction. Consider the dynamics of the error $\left(\tilde{R}, \tilde{b}_{g}\right) \in \mathrm{SO}(3) \times \mathbb{R}^{3}$ when resetting is applied. For each $\delta<$ $\delta^{*}:=c_{\mathrm{obs}}^{2}\left(1-\varepsilon^{2}\right)$, there is a $\tau^{*}(\delta)>0$, such that if $\tau$ is chosen so that $\tau \geq \tau^{*}(\delta)$, then for all initial conditions such that $\hat{R}(0) \in \mathrm{SO}(3)$ and $\hat{b}_{g}(0) \in \hat{\mathscr{B}}_{g}, \lim _{t \rightarrow \infty}\left\|\left((I-\tilde{R}), \tilde{b}_{g}\right)\right\|=0$.

Proof: The bias estimate remains bounded due to the parameter projection, and $\hat{R}$ remains bounded as it is confined to $\mathrm{SO}(3)$. We may write

$$
\begin{aligned}
J(\hat{R}) & =\frac{1}{2} \sum_{j=1}^{n}\left\|\bar{v}_{j}^{i}-\tilde{R} \bar{v}_{j}^{i}\right\|^{2}=\sum_{j=1}^{n}\left\|\bar{v}_{j}^{i}\right\|^{2}-\left(\bar{v}_{j}^{i}\right)^{\top} \tilde{R} \bar{v}_{j}^{i} \\
& =\sum_{j=1}^{n}-\left(\bar{v}_{j}^{i}\right)^{\top}\left(2 \tilde{s} S(\tilde{r})+2 S(\tilde{r})^{2}\right) \bar{v}_{j}^{i} \\
& =\sum_{j=1}^{n} 2\left\|\tilde{r} \times \bar{v}_{j}^{i}\right\|^{2} \geq c_{\mathrm{obs}}^{2}\|\tilde{r}\|^{2}=c_{\mathrm{obs}}^{2}\left(1-\tilde{s}^{2}\right) .
\end{aligned}
$$

Thus, if $|\tilde{s}|<\varepsilon$, then $J(\hat{R})>c_{\text {obs }}^{2}\left(1-\varepsilon^{2}\right)>\delta$. We also have $J(\hat{R}) \leq$ $2 n\|\tilde{r}\|^{2}$.

Consider the state of the observer at time $k \tau$ for some $k \in 1,2, \ldots$, and suppose first that $J(\hat{R}(k \tau)) \leq \delta$, so that no reset occurs at this time. By the above calculation, this implies that $|\tilde{s}(k \tau)| \geq \varepsilon$; that is, the state of the observer is within the region of attraction from Theorem 1, and hence the error will converge exponentially until the time $(k+1) \tau$. In particular, from the proof of Theorem 1, we may 
write

$$
\begin{aligned}
\left\|\left(\tilde{r}((k+1) \tau), \tilde{b}_{g}((k+1) \tau)\right)\right\|^{2} & \leq K^{2} \mathrm{e}^{-2 \lambda \tau}\left\|\left(\tilde{r}(k \tau), \tilde{b}_{g}(k \tau)\right)\right\|^{2} \\
& \leq K^{2} \mathrm{e}^{-2 \lambda \tau^{*}(\delta)}\left(1-\varepsilon^{2}+M^{2}\right) .
\end{aligned}
$$

Let $\tau^{*}(\delta)$ be chosen large enough that $K^{2} \mathrm{e}^{-2 \lambda \tau^{*}(\delta)}\left(1-\varepsilon^{2}+M^{2}\right)<$ $\delta /(2 n)$. Then $\|\tilde{r}((k+1) \tau)\|^{2}<\delta /(2 n)$, which implies by the above calculations that $J(\hat{R}((k+1) \tau)<\delta$. Thus, no reset occurs at time $(k+1) \tau$. By induction, no resets occur at any future point in time and the estimation error converges exponentially as indicated by Theorem 1. Suppose next that $J(\hat{R}(k \tau))>\delta$. In this case a reset occurs, so that after the reset, $\hat{R}=R^{*}=R$ and $|\tilde{s}|=1$. By the above analysis, the system now converges exponentially without any further resets.

As is clear from the proof of Theorem 2, the state of the observer is only reset if the estimates do not converge for some extended period of time. Thus, the resetting strategy should be viewed as an insurance policy against a lack of convergence-during normal operation, the observer works in the same way as the original observer without resetting, and it benefits from the complementary information contained in the vector observations and gyro measurements.

\section{Vi. Estimation of Vector Bias}

A potentially significant problem is the presence of bias in the vector measurements. We briefly consider a single biased vector measurement $v_{m}^{b}=v^{b}+b_{v}$ in the body-fixed frame, where $v^{b}$ is the true value of the vector and $b_{v}$ is a constant bias. By using the unbiased reference vector measurement $v_{m}^{i}=v^{i}=R v^{b}$, we shall construct an exponentially convergent estimator for $b_{v}$. The main trick is to compare the norm of $v_{m}^{b}$ and $v_{m}^{i}$, which should be equal if $v_{m}^{b}$ is bias-free. This technique has previously been used to construct several different estimators of magnetometer bias (see [25] for a survey).

Defining $y=\left\|v_{m}^{i}\right\|^{2}-\left\|v_{m}^{b}\right\|^{2}$ and $p=\left\|b_{v}\right\|^{2}$, and noting that $\left\|v^{i}\right\|=\left\|R v^{b}\right\|=\left\|v^{b}\right\|$, it is easily confirmed that $y=p-2\left(v_{m}^{b}\right)^{\top} b_{v}$. Defining the constant vector $\theta=\left[p, b_{v}^{\top}\right]^{\top}$ and the time-varying vector $\phi=\left[1,-2 v_{m}^{b}\right]^{\top}$, we have $y=\phi^{\top} \theta$. Based on this model, we may construct an over-parameterized observer

$$
\dot{\hat{\theta}}=\Gamma \phi\left(y-\phi^{\top} \hat{\theta}\right),
$$

where $\Gamma$ is a symmetric, positive-definite gain matrix. Defining the error variable $\tilde{\theta}=\theta-\hat{\theta}$, we can state the following result.

Theorem 3: Suppose that there exist $\varepsilon>0$ and $T>0$ such that, for each $t \geq 0, \int_{t}^{t+T} \phi(\tau) \phi^{\top}(\tau) \mathrm{d} \tau \geq \varepsilon I$. Then the origin is a globally exponentially stable equilibrium point for the error dynamics $\dot{\tilde{\theta}}=$ $-\Gamma \phi \phi^{\top} \tilde{\theta}$.

Proof: The proof can be completed, for example, by using the Lyapunov function $V=\frac{1}{2} \tilde{\theta}^{\top}\left(\Gamma^{-1}-\ell \int_{t}^{\infty} \mathrm{e}^{t-\tau} \phi(\tau) \phi(\tau)^{\top} \mathrm{d} \tau\right) \tilde{\theta}$, where $\ell>0$ is a small number, following previous results on parameter estimation (see, e.g., [26, Proposition 4]).

The requirement on $\phi$ in Theorem 3 is a standard PE condition that can be interpreted as requiring the components of $\phi$ to behave in a linearly independent manner when considered over sufficiently long time intervals. Since one of the components of $\phi$ is the constant 1 , this means that the three components of $v_{m}^{b}$ must be non-constant and vary independently of each other. Whether the PE requirement is reasonable or not depends on the application.

Remark 1: The model $y=\phi^{\top} \theta$ is a linear regression model with output $y$, regressor $\phi$, and parameter vector $\theta$. Several standard estimation methods can therefore be applied. For example, by introducing a time-varying gain $\Gamma$ satisfying $\dot{\Gamma}=\alpha \Gamma-\Gamma \phi \phi^{\top} \Gamma$, with $\alpha \geq 0$, we obtain the recursive least-squares estimate of $\theta$ with forgetting factor $\alpha$ [27, Th. 2.5].

The vector bias estimation can be combined with the attitude and gyro bias estimation, resulting in a cascaded structure. One of the previously available vectors $v_{j}^{b}$ is now replaced by a bias-corrected vector $v_{j}^{b}+b_{j v}-\hat{b}_{j v}=v_{j}^{b}+\tilde{b}_{j v}$. Since this vector may be close to zero when $\tilde{b}_{j v}$ is large, we replace the normalized vector $\bar{v}_{j}^{b}$ in the observer with $\bar{v}_{j}^{b *}:=\left(v_{j}^{b}+\tilde{b}_{j v}\right) / \max \left\{\left\|v_{j}^{b}+\tilde{b}_{j v}\right\|, \eta\right\}$, where $0<\eta \leq \underline{c}$ is a small number. It is also possible for the algebraic solution used in the resetting strategy to be ill-defined when $\tilde{b}_{j v}$ is large, despite Assumption 1 holding for the unbiased vectors; if this is the case, a reset is simply not performed. Both of these issues vanish after an initial transient, because $\tilde{b}_{j v}$ converges to zero. In order to analyze the behavior of the overall system, we need to assume that the algebraic solution $R^{*} \in \mathrm{SO}(3)$ is locally continuous, uniformly in $t$, with respect to small perturbations in the vector measurements. Such a property is easily proven for a simple algorithm such as TRIAD.

Theorem 4: Suppose that the vector bias estimation is combined with the attitude and gyro bias estimation. Under the conditions of Theorems 2 and $3, \lim _{t \rightarrow \infty}\left\|\left((I-\tilde{R}), \tilde{b}_{g}, \tilde{\theta}\right)\right\|=0$ for all initial conditions such that $\hat{R}(0) \in \operatorname{SO}(3), \hat{b}_{g}(0) \in \hat{\mathscr{B}}_{g}$, and $\hat{\theta}(0) \in \mathbb{R}^{4}$.

Proof (Outline): The error $\tilde{b}_{j v}$ vanishes as $t \rightarrow \infty$ so that $\bar{v}_{j}^{b *}$ approaches the unbiased vector $\bar{v}_{j}^{b}$. Let therefore $k$ be an integer chosen large enough that for all $t \geq k \tau,\left\|\bar{v}_{j}^{b *}-\bar{v}_{j}^{b}\right\| \leq \mu$, where $\mu>0$ is a constant to be determined. In particular, we choose $\mu$ small enough that $\left\|v_{j}^{b}+\tilde{b}_{j v}\right\| \geq \eta$ and that the algebraic solution used in the resetting strategy is well-defined.

Suppose first that $J(\hat{R}(k \tau)) \leq \delta$, so that no reset occurs at this time. Since $J(\hat{R})$ is continuous (uniformly in $t$ ), with respect to the vector measurements, we can show that there exists a $\mu_{1}$ such that if $\mu \leq \mu_{1}, J(\hat{R}) \leq \delta \Longrightarrow|\tilde{s}| \geq \varepsilon$. Since $\dot{V}$ is continuous (uniformly in $t$ ) with respect to the vector measurements, there exists a $\mu_{2}>0$ such that if $\mu \leq \mu_{2},|\tilde{s}|=\varepsilon \Longrightarrow \dot{V}<0$, and hence $|\tilde{s}|$ cannot become smaller than $\varepsilon$ before the time $t=(k+1) \tau$. The derivative $\dot{W}$ is also continuous (uniformly in $t$ ) with respect to the vector measurements, and hence we obtain the inequality $\dot{W} \leq-\alpha W+\rho_{\mu}(t)$, where $\rho_{\mu}(t)$ is a small perturbation with a bound that vanishes as $\mu \rightarrow 0$. Applying the comparison lemma we find that the trajectory of $W$ deviates from the unperturbed trajectory on the interval $[k \tau,(k+1) \tau]$ by an amount that can be made arbitrarily small by decreasing $\mu$. It is therefore straightforward to show that there exists a $\mu_{3}>0$ such that if $\mu \leq \mu_{3}$, $\|\tilde{r}((k+1) \tau)\|<\delta /(2 n)-v$, where $v>0$ is a small number. Again using continuity of $J(\hat{R})$, we can show that there exists a $\mu_{4}>0$ such that if $\mu \leq \mu_{4},\|\tilde{r}\|<\delta /(2 n)-v \Longrightarrow J(\hat{R})<\delta$. Hence, no reset occurs at time $t=(k+1) \tau$, and by induction, no resets occur at any future point in time. Again using $\dot{W} \leq-\alpha W+\rho_{\mu}(t)$ and noting that $\rho_{\mu}(t) \rightarrow 0$ as $t \rightarrow \infty$, we find that $W \rightarrow 0$, and therefore $\left((I-\tilde{R}), \tilde{b}_{g}\right) \rightarrow 0$ as $t \rightarrow \infty$. Next, suppose that $J(\hat{R}(k \tau))>\delta$, so that a reset occurs. Due to the local continuity of $R^{*}$, there exists a $\mu_{5}$ such that if $\mu \leq \mu_{5},|\tilde{s}| \geq \varepsilon$ after the reset. By the argument above, we therefore have $\left((I-\tilde{R}), \tilde{b}_{g}\right) \rightarrow 0$, as $t \rightarrow \infty$.

\section{EXAMPLE}

We illustrate the results of the note by using a recorded data set from a remotely controlled helicopter equipped with accelerometers and gyros (providing data at $100 \mathrm{~Hz}$ ), magnetometers $(10 \mathrm{~Hz})$, and a GPS receiver $(5 \mathrm{~Hz})$. Body-fixed vectors are provided by the magnetometers and accelerometers; reference vectors are provided by the earth's magnetic field and a filtered derivative of the GPS velocity (the latter being highly time-varying). We emphasize that this example is intended as an illustration based on a single data set, and not a performance evaluation.

The attitude and gyro bias observer is implemented at $100 \mathrm{~Hz}$ using forward Euler discretization, with tuning parameters $k_{1}=k_{2}=1$ and $k_{I}=0.2$. The attitude is maintained as a quaternion, which is normalized at each time step to compensate for numerical errors. 


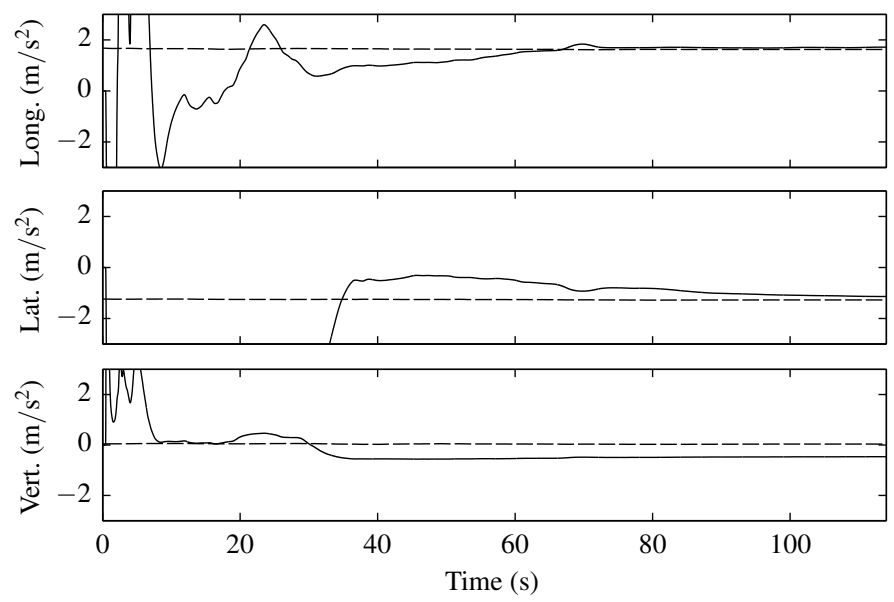

(a) Acceleration bias, EKF (dashed) and observer (solid)

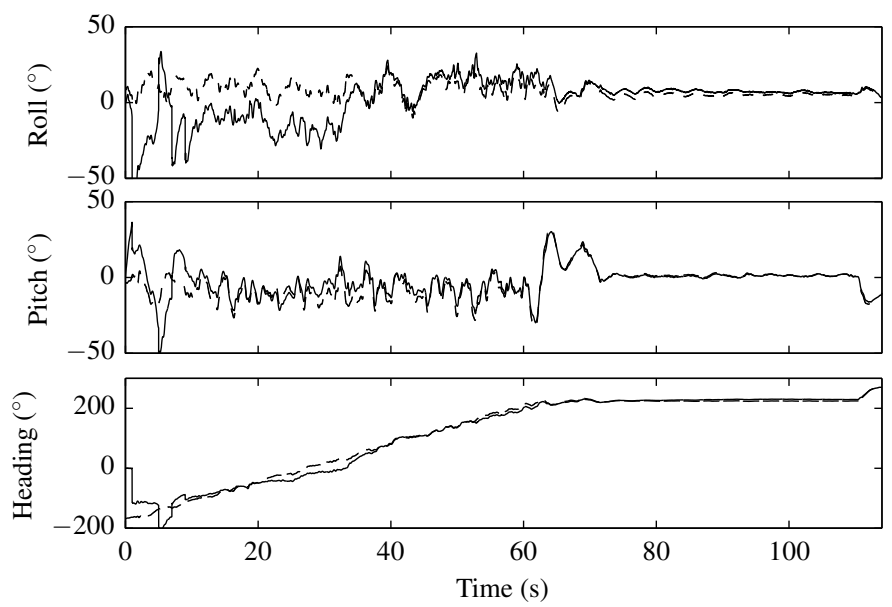

(c) Euler angles, EKF (dashed) and observer (solid)

Fig. 1. Results from helicopter example

The accelerometer bias is estimated using discrete-time recursive least squares, with $\Gamma(0)=10 I$. Resetting is implemented with $\tau=2 \mathrm{~s}$ and $\delta=0.4$ and the resetting value computed via QUEST.

Figs. 1(a)-(c) show the estimates of bias and attitude together with reference estimates from an EKF running online (no independent reference is available, and thus the purpose is not to benchmark the two approaches). Initially, the estimates are highly inaccurate, due primarily to very significant accelerometer biases, but also inaccurate initial values. Consequently, 4 resets occur within the first 10 seconds, which helps to improve the convergence time. The accelerometer biases in the EKF have been pre-calibrated through lab experiments and have had time to stabilize prior to the displayed time window. As the bias estimates improve, the estimates gradually fall into alignment with the EKF. To illustrate the benefit of integrating vector and gyro measurements, Fig. 1(d) shows the QUEST solution based on magnetometer measurements and bias-compensated accelerometer measurements (with the same reference vectors as in the observer). Much of the noise seen in the QUEST solution can be attenuated by more heavy low-pass filtering; however, this would come at the cost of increased phase lag.

\section{APPENDIX}

Let the set of possible parameters be defined by $\mathscr{B}_{g}:=\left\{b_{g} \in\right.$ $\left.\mathbb{R}^{3} \mid \mathscr{P}\left(b_{g}\right) \leq 0\right\}$, where $\mathscr{P}: \mathbb{R}^{3} \rightarrow \mathbb{R}$ is a smooth, convex function with gradient $\nabla \mathscr{P}^{\top}$. Let $\mathscr{B}_{g}^{0}$ denote the interior of $\mathscr{B}_{g}$,

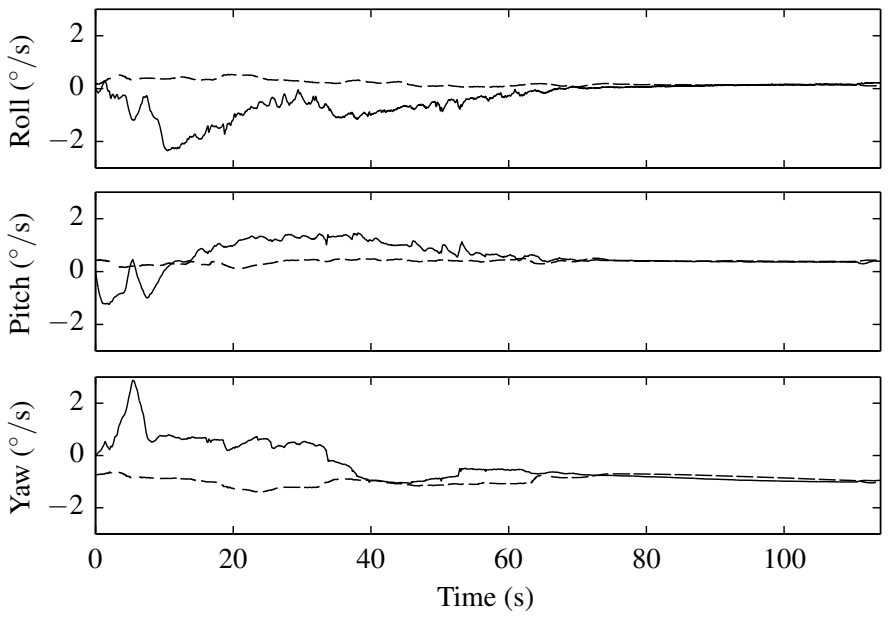

(b) Gyro bias, EKF (dashed) and observer (solid)

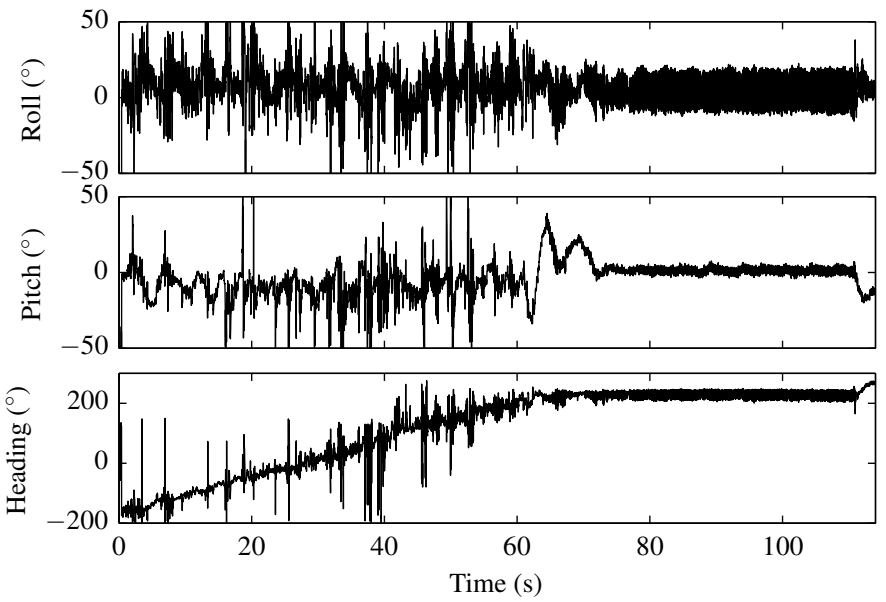

(d) Euler angles, QUEST

and let $\hat{\mathscr{B}}_{g}$ be defined by $\hat{\mathscr{B}}_{g}=\left\{\hat{b}_{g} \in \mathbb{R}^{3} \mid \mathscr{P}\left(\hat{b}_{g}\right) \leq \delta\right\}$, where $\delta$ is a small positive number, making $\hat{\mathscr{B}}_{g}$ a slightly larger superset of $\mathscr{B}_{g}$. Then $\operatorname{Proj}\left(\hat{b}_{g},-k_{I} \sigma\right)=p\left(\hat{b}_{g},-k_{I} \sigma\right)\left(-k_{I} \sigma\right)$, where $p\left(\hat{b}_{g},-k_{I} \sigma\right)=I$ if $\hat{b}_{g} \in \mathscr{B}_{g}^{0}$ or $\nabla \mathscr{P}^{\top} \cdot\left(-k_{I} \sigma\right) \leq 0$; and $p\left(\hat{b}_{g},-k_{I} \sigma\right)=$ $I-\min \left\{1, \mathscr{P}\left(\hat{b}_{g}\right) / \delta\right\} \nabla \mathscr{P} \nabla \mathscr{P}^{\top} /\|\nabla \mathscr{P}\|^{2}$ if $\hat{b}_{g} \in \hat{\mathscr{B}}_{g} \backslash \mathscr{B}_{g}^{0}$ and $\nabla \mathscr{P}^{\top}$. $\left(-k_{I} \sigma\right)>0$.

\section{ACKNOWLEDGMENTS}

The authors wish to thank E. Johnson, Georgia Institute of Technology, and H. Christophersen, Adaptive Flight Inc., for providing us with the helicopter data, and B. Vik, Norwegian University of Science and Technology, for many valuable discussions.

\section{REFERENCES}

[1] G. Wahba, "A least squares estimate of satellite attitude," SIAM Rev., vol. 8, no. 3, pp. 384-386, 1966.

[2] M. D. Shuster and S. D. Oh, "Three-axis attitude determination from vector observations," J. Guid. Contr. Dynam., vol. 4, no. 1, pp. 70-77, 1981.

[3] E. J. Lefferts, F. L. Markley, and M. D. Shuster, "Kalman filtering for spacecraft attitude estimation," in Proc. AIAA 20th Aerospace Sciences Meeting, Orlando, FL, 1982, pp. 1-16.

[4] J. L. Crassidis, F. L. Markley, and Y. Cheng, "Survey of nonlinear attitude estimation methods," J. Guid. Contr. Dynam., vol. 30, no. 1, pp. 12-28, 2007. 
[5] P. S. Maybeck, Stochastic Models, Estimation, and Control, Volume 1, ser. Mathematics in Science and Engineering. New York: Academic Press, 1979, vol. 141.

[6] R. Phillips and G. Schmidt, "GPS/INS integration," AGARD Lecture Series: System Implications and Innovative Applications of Satellite Navigation, vol. 207, pp. 9.1-9.18, 1996.

[7] S. Salcudean, "A globally convergent angular velocity observer for rigid body motion," IEEE Trans. Automat. Contr., vol. 36, no. 12, pp. 1493 1497, 1991.

[8] B. Vik and T. I. Fossen, "A nonlinear observer for GPS and INS integration," in Proc. IEEE Conf. Dec. Contr., Orlando, FL, 2001, pp. 2956-2961.

[9] J. Thienel and R. M. Sanner, "A coupled nonlinear spacecraft attitude controller and observer with an unknown constant gyro bias and gyro noise," IEEE Trans. Automat. Contr., vol. 48, no. 11, pp. 2011-2015, 2003.

[10] R. Mahony, T. Hamel, and J.-M. Pflimlin, "Nonlinear complementary filters on the Special Orthogonal Group," IEEE Trans. Automat. Contr., vol. 53, no. 5, pp. 1203-1218, 2008.

[11] T. Hamel and R. Mahony, "Attitude estimation on $\mathrm{SO}(3)$ based on direct inertial measurements," in Proc. IEEE Int. Conf. Robotics Automation, Orlando, FL, 2006, pp. 2170-2175.

[12] R. Mahony, T. Hamel, J. Trumpf, and C. Lageman, "Nonlinear observers on $\mathrm{SO}(3)$ for complementary and compatible measurements: A theoretical study," in Proc. IEEE Conf. Dec. Contr., Shanghai, China, 2009, pp. 6407-6412.

[13] M.-D. Hua, "Attitude estimation for accelerated vehicles using GPS/INS measurements," Contr. Eng. Pract., vol. 18, no. 7, pp. 723-732, 2010.

[14] J. C. Kinsey and L. L. Whitcomb, "Adaptive identification on the group of rigid-body rotations and its application to underwater vehicle navigation," IEEE Trans. Robot., vol. 23, no. 1, pp. 124-136, 2007.

[15] T. Lee, M. Leok, N. H. McClamroch, and A. Sanyal, "Global attitude estimation using single direction measurements," in Proc. American Contr. Conf., New York City, NY, 2007, pp. 3659-3664.

[16] J. F. Vasconcelos, C. Silvestre, and P. Oliveira, "A nonlinear observer for rigid body attitude estimation using vector observations," in Proc. IFAC World Congr., Seoul, South Korea, 2008, pp. 8599-8604.

[17] P. Batista, C. Silvestre, and P. J. Oliveira, "GES attitude observers - Part I: Multiple general vector observations," in Proc. IFAC World Congr, Milan, Italy, 2011, pp. 2985-2990.

[18] — "GES attitude observers - Part II: Single vector observations," in Proc. IFAC World Congr., Milan, Italy, 2011, pp. 2991-2996.

[19] H. F. Grip, A. Saberi, and T. A. Johansen, "Observers for cascaded nonlinear and linear systems," in Proc. IEEE Conf. Dec. Contr., Orlando, FL, 2011, pp. 3331-3337.

[20] - "Observers for interconnected nonlinear and linear systems," Automatica, 2012, to appear.

[21] H. F. Grip, T. I. Fossen, T. A. Johansen, and A. Saberi, "Attitude estimation based on time-varying reference vectors with biased gyro and vector measurements," in Proc. IFAC World Congr., Milan, Italy, 2011, pp. 8497-8502.

[22] T. I. Fossen, Handbook of Marine Craft Hydrodynamics and Motion Control. Wiley, 2011.

[23] M. Krstić, I. Kanellakopoulos, and P. V. Kokotović, Nonlinear and Adaptive Control Design. New York: Wiley, 1995.

[24] H. K. Khalil, Nonlinear Systems, 3rd ed. Upper Saddle River, NJ: Prentice-Hall, 2002.

[25] R. Alonso and M. D. Shuster, "Attitude-independent magnetometer bias determination: A survey," J. Astronaut. Sci., vol. 50, no. 4, pp. 453-475, 2002.

[26] H. F. Grip, T. A. Johansen, L. Imsland, and G.-O. Kaasa, "Parameter estimation and compensation in systems with nonlinearly parameterized perturbations," Automatica, vol. 46, no. 1, pp. 19-28, 2010.

[27] K. J. Åström and B. Wittenmark, Adaptive Control, 2nd ed. Reading, MA: Addison-Wesley, 1995. 